\title{
Distinct propagating fast wave trains associated with flaring energy releases
}

\author{
D. Yuan ${ }^{1}$, Y. Shen ${ }^{2}$, Y. Liu ${ }^{2}$, V. M. Nakariakov ${ }^{1,3}$, B. Tan ${ }^{4}$, and J. Huang ${ }^{4}$ \\ ${ }^{1}$ Centre for Fusion, Space and Astrophysics, Department of Physics, University of Warwick, Coventry CV4 7AL, UK \\ e-mail: Ding. Yuan@warwick.ac.uk \\ 2 Yunnan Astronomical Observatory, Chinese Academy of Sciences, PO Box 110, Kunming 650011, PR China \\ ${ }^{3}$ Central Astronomical Observatory at Pulkovo of the Russian Academy of Sciences, 196140 St Petersburg, Russia \\ ${ }^{4}$ Key Laboratory of Solar Activity, National Astronomical Observatories, Chinese Academy of Sciences, Beijing 100012, PR China
}

Received 8 March 2013 / Accepted 3 May 2013

\section{ABSTRACT}

\begin{abstract}
Context. Large-scale fast waves with perturbation of the EUV emission intensity are well resolved in both temporal and spatial scale by SDO/AIA. These waves are prone to propagate along the magnetic field line.

Aims. We aim to probe the link between propagating fast wave trains and flaring energy releases. By measuring the wave parameters, we reveal their nature and investigate the potential to diagnose the energy source and waveguide.

Methods. The spatial and temporal evolution of the wave amplitude and propagating speed are studied. The correlation of individual wave trains with flare-generated radio bursts is tested.

Results. The propagating wave pattern comprises distinct wave trains with varying periods and wavelengths. This characteristic signature is consistent with the patterns formed by waveguide dispersion, when different spectral components propagate at different phase and group speeds. The wave train releases are found to be highly correlated in start time with the radio bursts emitted by the nonthermal electrons that were accelerated in bursty energy releases. The wave amplitude is seen to reach the maximum midway during its course. This can be caused by a combined effect of the waveguide spread in the transverse direction and density stratification. The transverse amplitude distribution perpendicular to the wave vector is found to follow approximately a Gaussian profile. The spatial structure is consistent with the kink mode that is polarised along the line-of-sight. The propagating speed is subject to deceleration from $\sim 735-845 \mathrm{~km} \mathrm{~s}^{-1}$ to $\sim 600 \mathrm{~km} \mathrm{~s}^{-1}$. This could be caused by the decrease in the local Alfvén speed and/or the projection effect.
\end{abstract}

Key words. Sun: atmosphere - Sun: corona - Sun: UV radiation - Sun: oscillations - Sun: flares - Sun: radio radiation

\section{Introduction}

The theory of magnetohydrodynamic (MHD) waves in structured plasma was developed in the 1970s and 1980s (e.g., Zajtsev \& Stepanov 1975; Roberts 1981b,a; Edwin \& Roberts 1982, 1983). During the past decades, various MHD wave modes were confidently detected with modern instruments and were exploited for seismological applications (see reviews by Nakariakov et al. 2005; De Moortel \& Nakariakov 2012). Standing fast kink waves were detected in the closed coronal loops (e.g. Nakariakov et al. 1999; Aschwanden et al. 1999; White et al. 2012) and in post-flare arcades (Verwichte et al. 2005). The global (fundamental) kink mode was implemented to estimate the magnetic field strength (so called MHD seismology, e.g. Nakariakov \& Ofman 2001). Propagating fast kink waves were found in the coronal loops (Williams et al. 2002; Van Doorsselaere et al. 2008). Trapped fast sausage modes, due to the existence of the cut-off wavenumber (Nakariakov et al. 2003), are only supported by sufficiently thick and dense loops. Some spatially-resolved radio imaging observations were consistent with the features of fast sausage waves (e.g. Asai et al. 2001; Melnikov et al. 2005). Standing slow mode waves were detected as intensity and Doppler-velocity oscillations in the post-flare loops using spectrometric data (Wang et al. 2003). Propagating slow mode waves were confidently detected near the footpoints of active region loops (e.g., De Moortel et al. 2000; Marsh et al. 2009; Wang et al. 2009; Verwichte et al. 2010; Yuan \& Nakariakov 2012).
In the SDO/AIA era (Lemen et al. 2012; Boerner et al. 2012), the full solar disk is recorded with high temporal and fine spatial resolution. It allows for confident detection of sporadically occurring coronal fast magneto-acoustic waves (Liu et al. 2011; Shen \& Liu 2012). It was found that quasi-periodic propagating fast waves exhibit similar periodicities (with the most prominent peak at about $3 \mathrm{~min}$ ) with the quasi-periodic pulsations (QPP) in X-ray emission (Liu et al. 2011; Ofman et al. 2011). This result is consistent with previous studies, where 3 min oscillations are sourced to the chromospheric level in sunspot umbrae and found to modulate QPP in the form of slow mode MHD waves (Sych et al. 2009). Flares are also known to generate non-thermal electrons that are responsible for microwave and $\mathrm{X}$-ray emissions. Moreover, flares can excite MHD oscillations. The relationship of QPP in microwave and X-ray emission and coronal MHD waves was intensively investigated (see review by Nakariakov \& Melnikov 2009).

It was demonstrated that an impulsively-generated fast magneto-acoustic wave at a distance away from the source develops into a quasi-periodic wave train with a pronounced frequency and amplitude modulation owing to the dispersion properties of guided fast waves, if it is guided by a region of low Alfvén speed (i.e., coronal loop, current sheet; Roberts et al. 1984; Murawski \& Roberts 1994). The time signature shows a very typical tadpole wavelet spectrum: a narrow-spectrum long-period head is followed by a broad-spectrum short-period tail (Nakariakov et al. 2004). However, this tadpole wavelet 
spectrum is only prominent if the initial spectrum is sufficiently broad and situates mostly above the cut-off wavenumber (Nakariakov et al. 2005). The radio emissions associated with solar flares were also found to be modulated by propagating fast waves and exhibit evident tadpole wavelet spectra (Mészárosová et al. 2009a,b).

In this paper, we report the observation of distinct quasiperiodic propagating fast magneto-acoustic wave trains (QPF). The QPF processes are described in Sect. 2. The analysis and results are presented in Sect. 3. The discussion and conclusion are given in Sect. 4.

\section{Observations}

We report an observational study of a QPF wave event on 30 May 2011. A GOES C2.8 flare occurred at 10:48 UT in the active region AR11227. Propagating fast magneto-acoustic wave trains were observed as intensity perturbations at a distance of about $110 \mathrm{Mm}$ from the flare epicentre at 10:50:12 UT. They are only detected in the AIA $171 \AA$ bandpass. The lag time between the flare occurring and the first wavefront appearing was about $2.2 \mathrm{~min}$. The average energy transit speed was estimated at about $833 \mathrm{~km} \mathrm{~s}^{-1}$, which was well in the Alfvén (fast) wave speed range in the coronal condition. The data preparation and initial analysis were presented in Shen \& Liu (2012). A shorter data set for about 26 min was used, starting from 10:45 UT. The data cube is a set of images with the size of $834 \times 834$ pixels. The images were normalised with its exposure time and were interpolated into a uniform time grid with a $12 \mathrm{~s}$ cadence.

It was shown that the GOES and RHESSI light curves exhibit a very steady increase in the particle counts during the flare. No fine structure of flare emission was significantly detected in soft and hard X-ray bands (Shen \& Liu 2012). To show the fine structure of the flare pulsations, we obtained the radio observation in the same time interval (10:45-11:11 UT) at $173.2 \mathrm{MHz}$ and $228.0 \mathrm{MHz}$, provided by the Nançay Radioheliograph (NRH, cf. Fig. 2, top panel). It reflects the radio emission of the flare-accelerated non-thermal electrons.

\section{Analysis and results}

We identify three distinct wave trains in this QPF event, which exhibit different properties: (We denote the wave trains as Train-1 or T1, Train-2 or T2, and Train-3 or T3. cf. Fig. 1):

1. There are obvious gaps in space (time) between the leading and trailing trains with a value of about $40.7 \mathrm{Mm}(72 \mathrm{~s})$ between $\mathrm{T} 1$ and $\mathrm{T} 2$, and a value of about $35.0 \mathrm{Mm}(90 \mathrm{~s})$ between $\mathrm{T} 2$ and $\mathrm{T} 3$.

2. The wavelengths (periods) are quite different: $33.7 \pm 0.6 \mathrm{Mm}$ $(58 \pm 1 \mathrm{~s}), 24.4 \pm 0.1 \mathrm{Mm}(40 \pm 0.5 \mathrm{~s})$, and $25.7 \pm 0.3 \mathrm{Mm}$ $(38 \pm 1 \mathrm{~s})$ for $\mathrm{T} 1, \mathrm{~T} 2$, and $\mathrm{T} 3$, respectively.

3. The wave trains exhibit different initial phase speeds: $735 \pm$ $58 \mathrm{~km} \mathrm{~s}^{-1}$ (T1), $845 \pm 42 \mathrm{~km} \mathrm{~s}^{-1}$ (T2) and $820 \pm 90 \mathrm{~km} \mathrm{~s}^{-1}$ (T3).

4. The wave trains agree in arising time with radio bursts generated by the non-thermal electrons (cf. Fig. 2, top panel).

Therefore, we divide the QPF into three wave trains and measure their parameters separately.

We first look at how the wave trains are correlated with the flaring radio bursts. It was found that the frequency of radio emissions in the magnetic reconnection site and energy releasing

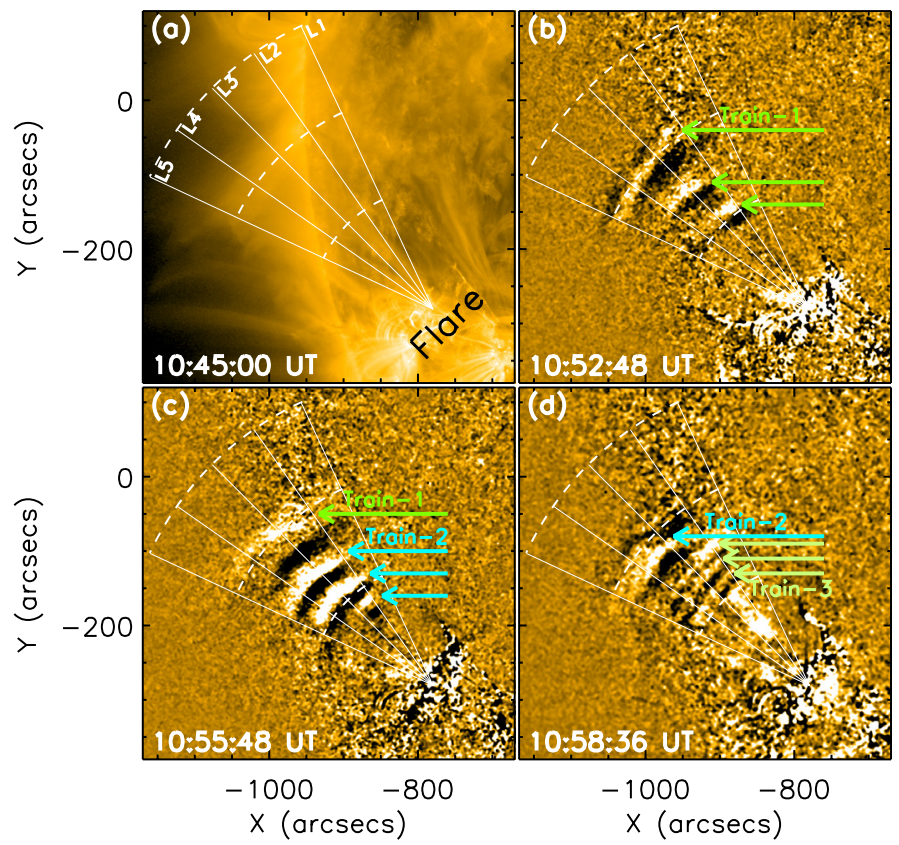

Fig. 1. a) The AIA field-of-view in $171 \AA$, displaying the active region AR11227 and the flare epicenter. b)-d) Running difference images illustrate the QPF wave trains. The wavefronts of three distinct wave trains are marked by arrow groups in varied colors. The solid lines (L1-L5) forming a fan geometry are the cuts used in making timedistance arrays. The dashed arcs are concentric at the flare epicenter, the radii are $100 \mathrm{Mm}, 200 \mathrm{Mm}$ and $300 \mathrm{Mm}$, respectively.

region are in the range of 400-1000 $\mathrm{MHz}$ (Bastian et al. 1998). The radio emission at $173.2 \mathrm{MHz}$ is sourced at a height lower than $228.0 \mathrm{MHz}$, but higher than both the magnetic reconnection site and energy releasing region (Bastian et al. 1998). This explains the time lag between the $\mathrm{NRH}$ radio bursts and the flare start time. The flare started at 10:48 UT. The first radio burst arose at about 10:49:12 UT at the $228.0 \mathrm{MHz}$ band. Train-1 started at about $110 \mathrm{Mm}$ from the flare epicenter at about 10:50:12 UT. The radio emissions at 173.2 MHz peaked at about 10:50:30 UT, and slightly lagged behind Train-1. The second radio burst appeared between 10:53 and 10:55 UT, first appearing at 228.0 MHz, and then at $173.2 \mathrm{MHz}$. Train-2 started at 10:54:24 UT about $1 \mathrm{~min}$ after the burst at $228.0 \mathrm{MHz}$. The third radio burst appeared between 10:57 and 11:00 UT, only at 173.2 MHz. No significant burst was detected at 228.0 MHz. The start time of Train-3 was at 10:58:12 UT during the third radio burst. We infer that at least three flaring energy pulses were released during the flare. The flaring energy releases apparently triggered the fast wave trains. Non-thermal electrons were concurrently accelerated and transported to a higher atmosphere, and then generated the radio bursts. It is logical that the fast wave trains and radio bursts exhibit high correlations at the start times.

The dispersive evolution of impulsively-generated fast wave trains is determined by the density profile of the waveguide. The time signature of the wave train displays a characteristic tadpole wavelet spectrum, exhibiting a gradually decreasing period at a fixed height and hence, a stretching wavelength along the waveguide at a fixed time (Nakariakov et al. 2004). The time series of the amplitude variation at about $150 \mathrm{Mm}$ from the flare epicenter (Fig. 2, bottom panel) shows that the oscillating periods of Train- 1 and -2 decrease at the observational height. This feature 
D. Yuan et al.: fast wave
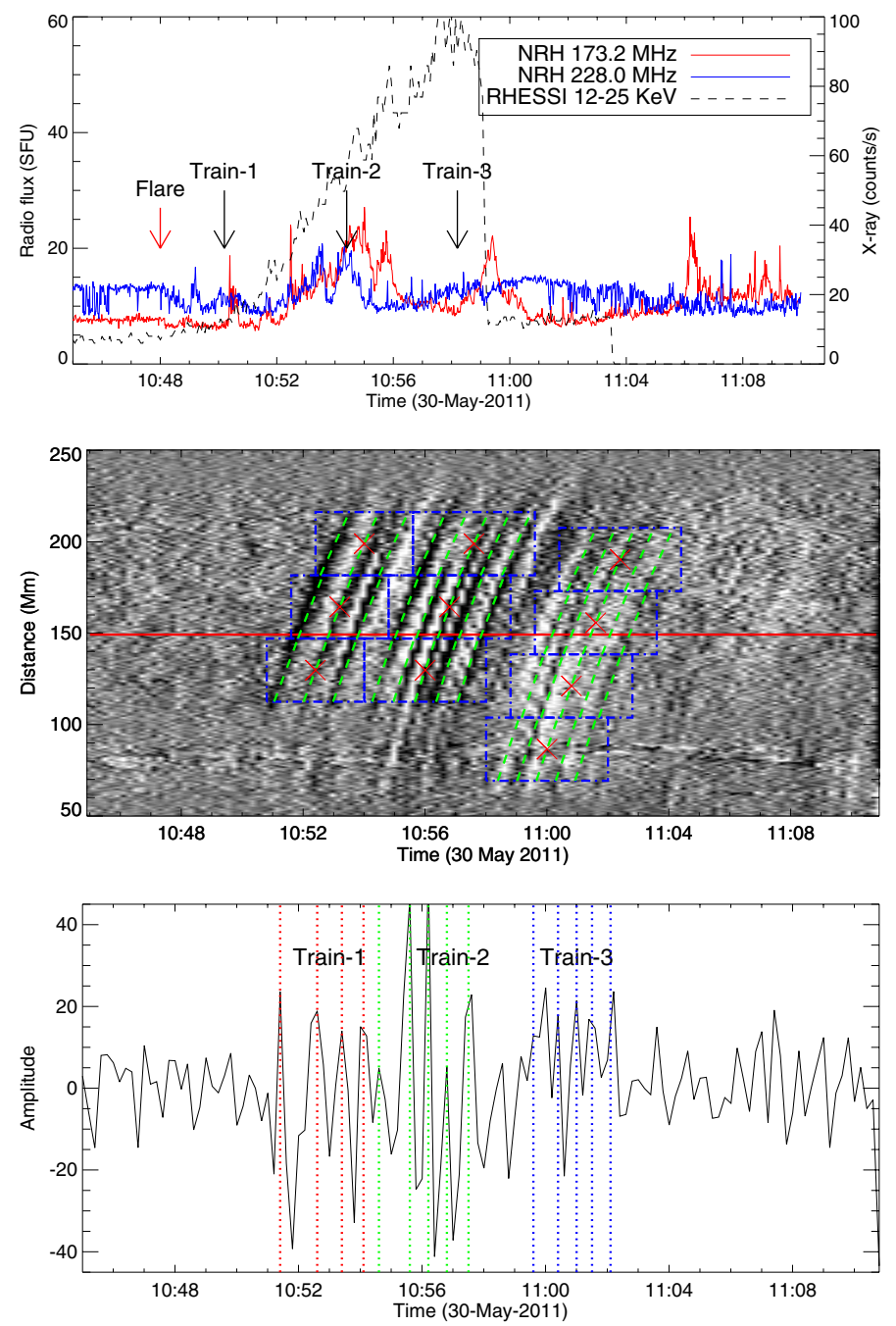

Fig. 2. Top panel: the radio emission of AR11227 in NRH $173.2 \mathrm{MHz}$ and $228.0 \mathrm{MHz}$ band and the RHESSI hard X-ray count rate in $12-25 \mathrm{KeV}$. The start time of the flare, Train-1, Train-2 and Train-3 are labeled at the time axis. Middle panel: the time-distance plot for L3. The samples used to measure the phase speed are enclosed in blue rectangles, the centers are labeled in red cross. The measured results are over-plotted within each rectangle as dashed curves. Bottom panel: the amplitude variation at a fixed height of about $150 \mathrm{Mm}$ (marked in red line in the middle panel). The dotted lines mark approximately the wave cycles of three wave trains in varied color.

is in agreement with the dispersively evolving wave (Nakariakov et al. 2005), given the limited number of wave fronts and the coarse cadence compared to the wave period (cf. Table 1). This effect is not so pronounced for Train-3. Its amplitude is lower than that of Train-1 and -2 , and its signal is more affected by the noise. In Fig. 3, we display the spatial variation in the wavelengths of the wave trains along L3. It is shown that the wavelengths stretch along the open coronal loops. Besides a decreasing period, it is another indicator of dispersive evolution of the fast wave trains. The increasing wave length could also be caused by an accelerating phase speed or an increasing oscillating period. However, both the oscillating periods and the following speed measure explicitly exhibit the opposite behaviour, and hence, we rule out this alternative interpretation. The time signature and wavelength of QPF indicates that the fast magneto-acoustic wave is likely to be triggered by an impulsive energy source. It is not possible to diagnose the loop density

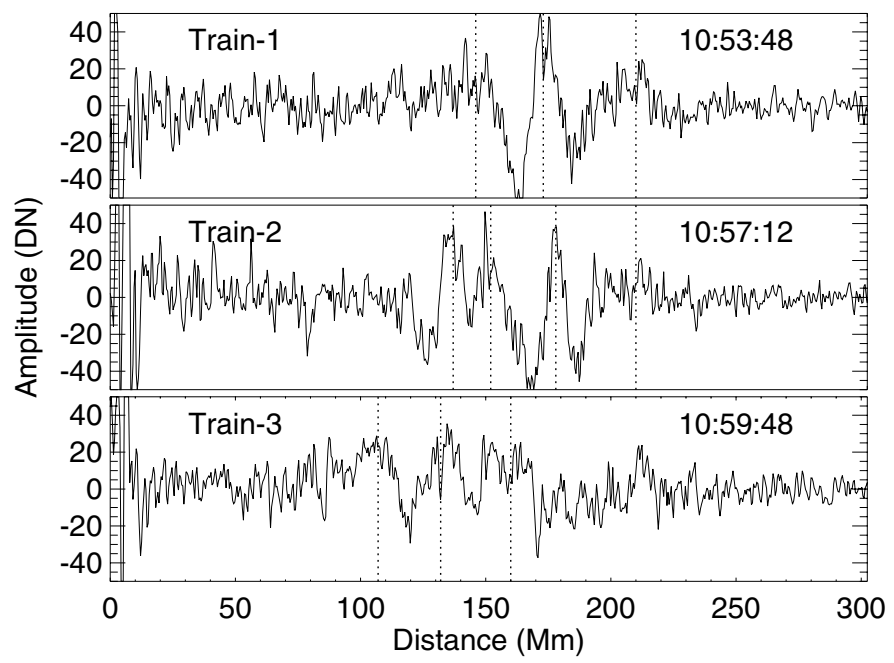

Fig. 3. Instant cuts along L3 at the fixed times that display the wavelength during the wave train evolutions. The horizontal axis denotes the distance along the wave path. The observation times for each snapshot are shown in the panels. The vertical dotted lines mark the approximate positions of the wave fronts.

profile at the current stage as suggested by Nakariakov et al. (2004), because of the limited temporal resolution of modern instruments.

We used the best similarity measure method (BSM, Yuan \& Nakariakov 2012) to obtain the phase speed and estimate its error (Fig. 2, middle panel). The BSM seeks for the best (minimum) similarity (see e.g. Santini \& Jain 1999) between a propagating wave model and the time-distance plot. The period and phase speed are allowed to vary within a certain range, as long as the similarity measure remains within $1 \%$ above its minimun value. This gives good estimates of the error bars (Yuan \& Nakariakov 2012). Figure 4 (bottom panel) shows that the wave trains started with slightly different initial phase speeds: $735 \pm 58 \mathrm{~km} \mathrm{~s}^{-1}$ (T1), $845 \pm 42 \mathrm{~km} \mathrm{~s}^{-1}$ (T2), and $820 \pm$ $90 \mathrm{~km} \mathrm{~s}^{-1}$ (T3). However, they appeared to end with the same projected phase speed at about $600 \mathrm{~km} \mathrm{~s}^{-1}$. The deceleration was found to be $1.35 \pm 0.67 \mathrm{~km} \mathrm{~s}^{-2}, 2.27 \pm 0.39 \mathrm{~km} \mathrm{~s}^{-2}$, and $1.31 \pm 0.56 \mathrm{~km} \mathrm{~s}^{-2}$ for $\mathrm{T} 1, \mathrm{~T} 2$ and $\mathrm{T} 3$, respectively.

Figure 4 (top panel) illustrates that the wave amplitudes increase with distance, then peak, and finally then decay gradually. This effect is very prominent in Train-2: it reaches a maximum amplitude at about $180 \mathrm{Mm}$, which is about $21 \%$ larger than the value at about $130 \mathrm{Mm}$.

The amplitude profile normal to the wave vector is also resolved with SDO/AIA. A wave front evolution of Train-2 is displayed in Fig. 5. The transverse distribution of the wave amplitude was approximated with a Gaussian profile. It shows that the wave front extends gradually along the waveguiding structure. It was found that the density perturbations of a kink (sausage) wave inside and outside a coronal loop exhibit in-phase (outof-phase) motion (Cooper et al. 2003b). The wave propagation observed as intensity perturbations inside and outside the coronal loop are in-phase in our case. This spatial structure is more consistent with the kink mode polarised along the line-of-sight. In this case, the kink mode can be observed as emission intensity variations because of its modulation to the line-of-sight column depth of the oscillating plasma structure (Cooper et al. 2003a). 

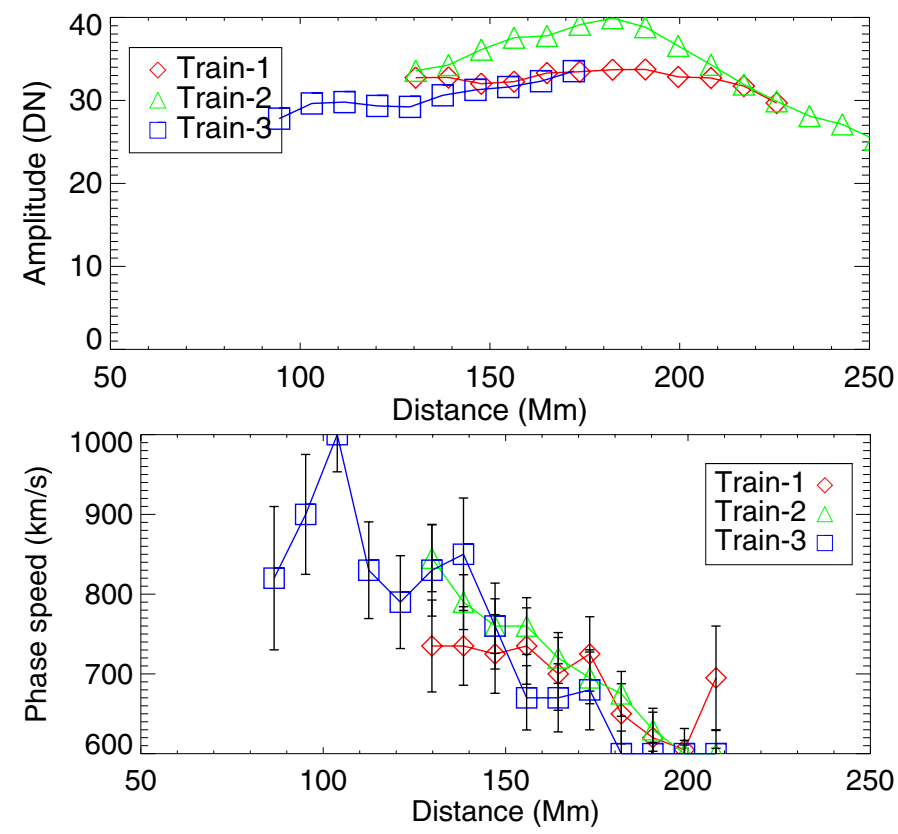

Fig. 4. Wave amplitude (top) and projected phase speed (bottom) are plotted as a function of the distance to the flare epicenter for the wave trains. The red diamonds, green triangles, and blue squares denote the parameters of Train-1, -2 , and -3 , respectively.

\section{Discussion and conclusion}

In this work, we observed a QPF event with distinct wave trains. The wave trains were found to correlate in start time with the radio bursts observed with NRH. The wave fronts did not appear at the flare epicenter but rather a distance of about 70-110 Mm from it. No significant intensity perturbation was detected inbetween. This behaviour is consistent with the interpretation in terms of the kink mode, provided the waveguide is of a loop shape. In this case, the kink mode can become visible only in the segment of the loop that has a preferable angle to the line-ofsight (Cooper et al. 2003a).

The wave trains are found to correlate well in start time with radio bursts observed with NRH (Fig. 2, top panel). The wave trains and the non-thermal electrons (radio burst) apparently originate in the same energy release. The observed fast wave trains have wavelength modulation: components with longerwavelength propagate faster. This behaviour is consistent with the dispersion of fast magneto-acoustic waves in field-aligned plasma waveguides (Nakariakov et al. 2004).

The projected propagating phase speed was found to be in the Alfvén speed range (about 735-845 $\mathrm{km} \mathrm{s}^{-1}$ ). The speed difference reaches about $15 \%$ for different wave trains at the start position, but the wave trains end with the same phase speed at about $600 \mathrm{~km} \mathrm{~s}^{-1}$. The deceleration was found to be $1.35 \pm$ $0.67 \mathrm{~km} \mathrm{~s}^{-2}, 2.27 \pm 0.39 \mathrm{~km} \mathrm{~s}^{-2}$, and $1.31 \pm 0.56 \mathrm{~km} \mathrm{~s}^{-2}$ for $\mathrm{T} 1$, $\mathrm{T} 2$, and $\mathrm{T} 3$, respectively. The deceleration may be caused by the decrease in the Alfvén speed (a joint effect of the magnetic flux tube divergence and stratified density along the loops). However, it may also be a contribution of projection effect.

The QPF wave amplitudes were found to amplify during its passage, and then started to decay midway (Fig. 4, top panel). The wave amplitude is determined by both the wave energy and the properties of the waveguide, such as in the density stratification. As the wave propagates along the open coronal structure, the wave energy spreads to a broader extent. Therefore, the wave amplitude tends to decrease. The density stratification
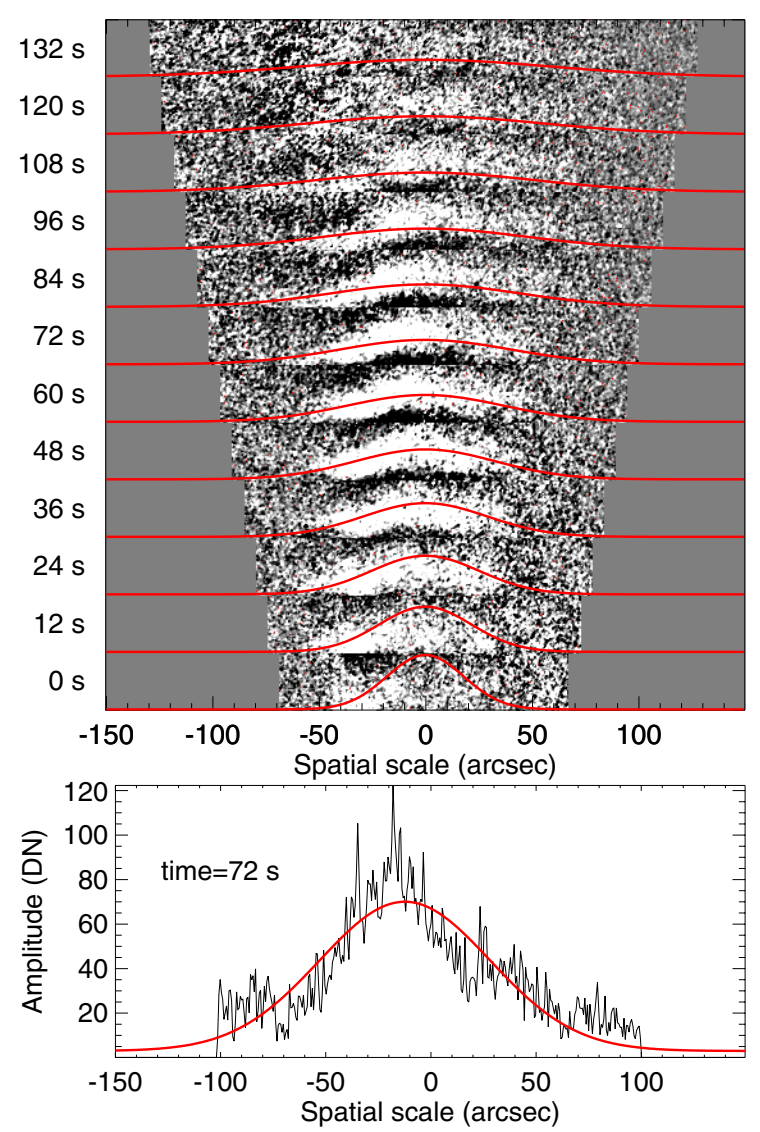

Fig. 5. Top panel: wave front evolution of Train-2 as a function of time. The red curves are Gaussian profiles. The linearly increasing widths are adjusted manually. Bottom panel: an example (72 s) of the crosssectional distribution of amplitude and the Gaussian fit (red curve).

Table 1. Observables and physical parameters of the QPF wave trains.

\begin{tabular}{lccc}
\hline \hline Parameters & Train-1 & Train-2 & Train-3 \\
\hline Start time (UT) & $10: 50: 12$ & $10: 54: 24$ & $10: 58: 12$ \\
End time (UT) & $10: 56: 12$ & $10: 59: 12$ & $11: 02: 36$ \\
Start position (Mm) & $\sim 110$ & $\sim 80$ & $\sim 70$ \\
Spatial lag (Mm) & $\ldots$ & $\sim 40.7$ & $\sim 35.0$ \\
Time lag (s) & $\ldots$ & $\sim 72$ & $\sim 90$ \\
Wavefront number & 3 & 5 & $\sim 5$ \\
Wavelength (Mm) & $33.7 \pm 0.5$ & $24.4 \pm 0.1$ & $25.7 \pm 0.3$ \\
Period (s) & $58 \pm 1$ & $40 \pm 0.5$ & $38 \pm 1$ \\
Deceleration $\left(\mathrm{km} \mathrm{s}^{-2}\right)$ & $1.35 \pm 0.67$ & $2.27 \pm 0.39$ & $1.31 \pm 0.56$ \\
\hline
\end{tabular}

along the loops are prone to amplify the wave amplitude. These two factors can lead to the appearance of a maximum amplitude midway.

The amplitude distribution normal to the wave vector follows a Gaussian profile. This spatial structure is prescribed by the kink eigenmode of the waveguide. Moreover, the wave propagation inside and outside the loop exhibit in-phase collective motions. This feature is more consistent with fast kink wave.

The observation of fast magneto-acoustic waves provides useful information on the connectivity with flare pulsations. The fine measurements of its wave parameters reflect the details of the plasma and magnetic field. It is a potential tool to probe the physical parameters. However, numerical simulations are required to fully understand the wave generation, propagation, and decay. 
Acknowledgements. The work is supported by the Marie Curie PIRSES-GA2011-295272 RadioSun project, the European Research Council under the SeismoSun Research Project No. 321141 (DY, VMN), the Natural Science Foundation of China Grant No. 11073050, the grant 8524 of the Ministry of Education and Science of the Russian Federation (VMN), and the Kyung Hee University International Scholarship (VMN).

\section{References}

Asai, A., Shimojo, M., Isobe, H., et al. 2001, ApJ, 562, L103

Aschwanden, M. J., Fletcher, L., Schrijver, C. J., \& Alexander, D. 1999, ApJ, 520,880

Bastian, T. S., Benz, A. O., \& Gary, D. E. 1998, ARA\&A, 36, 131

Boerner, P., Edwards, C., Lemen, J., et al. 2012, Sol. Phys., 275, 41

Cooper, F. C., Nakariakov, V. M., \& Tsiklauri, D. 2003a, A\&A, 397, 765

Cooper, F. C., Nakariakov, V. M., \& Williams, D. R. 2003b, A\&A, 409, 325

De Moortel, I., \& Nakariakov, V. M. 2012, Roy. Soc. London Philos. Trans. Ser. A, 370,3193

De Moortel, I., Ireland, J., \& Walsh, R. W. 2000, A\&A, 355, L23

Edwin, P. M., \& Roberts, B. 1982, Sol. Phys., 76, 239

Edwin, P. M., \& Roberts, B. 1983, Sol. Phys., 88, 179

Lemen, J. R., Title, A. M., Akin, D. J., et al. 2012, Sol. Phys., 275, 17

Liu, W., Title, A. M., Zhao, J., et al. 2011, ApJ, 736, L13

Marsh, M. S., Walsh, R. W., \& Plunkett, S. 2009, ApJ, 697, 1674

Melnikov, V. F., Reznikova, V. E., Shibasaki, K., \& Nakariakov, V. M. 2005, A\&A, 439, 727

Mészárosová, H., Karlický, M., Rybák, J., \& Jiřička, K. 2009a, A\&A, 502, L13

Mészárosová, H., Karlický, M., Rybák, J., \& Jiřička, K. 2009b, ApJ, 697, L108
Murawski, K. \& Roberts, B. 1994, Sol. Phys., 151, 305

Nakariakov, V. M., \& Melnikov, V. F. 2009, Space Sci. Rev., 149, 119

Nakariakov, V. M., \& Ofman, L. 2001, A\&A, 372, L53

Nakariakov, V. M., Ofman, L., Deluca, E. E., Roberts, B., \& Davila, J. M. 1999, Science, 285,862

Nakariakov, V. M., Melnikov, V. F., \& Reznikova, V. E. 2003, A\&A, 412, L7

Nakariakov, V. M., Arber, T. D., Ault, C. E., et al. 2004, MNRAS, 349, 705

Nakariakov, V. M., Pascoe, D. J., \& Arber, T. D. 2005, Space Sci. Rev., 121, 115

Ofman, L., Liu, W., Title, A., \& Aschwanden, M. 2011, ApJ, 740, L33

Roberts, B. 1981a, Sol. Phys., 69, 39

Roberts, B. 1981b, Sol. Phys., 69, 27

Roberts, B., Edwin, P. M., \& Benz, A. O. 1984, ApJ, 279, 857

Santini, S., \& Jain, R. 1999, Pattern Analysis and Machine Intelligence, IEEE Trans., 21, 871

Shen, Y., \& Liu, Y. 2012, ApJ, 753, 53

Sych, R., Nakariakov, V. M., Karlicky, M., \& Anfinogentov, S. 2009, A\&A, 505, 791

Van Doorsselaere, T., Nakariakov, V. M., \& Verwichte, E. 2008, ApJ, 676, L73

Verwichte, E., Nakariakov, V. M., \& Cooper, F. C. 2005, A\&A, 430, L65

Verwichte, E., Marsh, M., Foullon, C., et al. 2010, ApJ, 724, L194

Wang, T. J., Solanki, S. K., Innes, D. E., Curdt, W., \& Marsch, E. 2003, A\&A, 402, L17

Wang, T. J., Ofman, L., \& Davila, J. M. 2009, ApJ, 696, 1448

White, R. S., Verwichte, E., \& Foullon, C. 2012, A\&A, 545, A129

Williams, D. R., Mathioudakis, M., Gallagher, P. T., et al. 2002, MNRAS, 336, 747

Yuan, D., \& Nakariakov, V. M. 2012, A\&A, 543, A9

Zajtsev, V. V., \& Stepanov, A. V. 1975, Issledovaniia Geomagnetizmu Aeronomii i Fizike Solntsa, 37, 3 